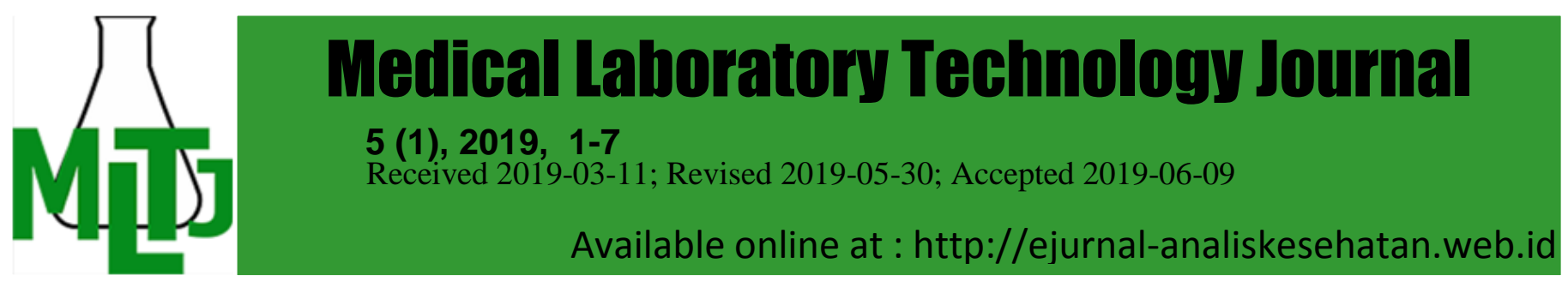

\title{
Association of the TG/HDL-C and Monocyte Count/HDL-C Ratios with Coronary Artery Disease in Non-ST Segment Elevation Myocardial Infarction
}

\author{
*Küçük Uğur, Kurt Recep \\ Sivas numune state hospital, Cardiology. Sivas, Turkey. \\ *Email: :drugurkucuk@hotmail.com \\ DOI: 10.31964/mltj.v5i1.207
}

\begin{abstract}
Monocyte Count/HDL ratio (MHR) is a simple and inexpensive parameter used as a risk predictor in patients diagnosed with myocardial infarction (MI). This study aims to investigate the association between Triglyceride/HDL-C (TG/HDL-C) ratio and coronary artery disease in patients diagnosed with non-ST segment elevation MI (NSTEMI). Total of 100 patients, 50 patients with NSTEMI and 50 healthy individuals, who had admitted to the cardiology outpatient clinic between January 2018 and January 2019 included in the study. Higher monocyte count, $\mathrm{MHR}$, and TG/HDL-C ratios detected in patients with coronary artery disease in comparison to healthy individuals. Although monocyte count was higher in NSTEMI group, the difference was not statistically significant $(p=0.101)$. There was an increase in the MHR NSTEMI group when compared against the control group $(p<0.05)$. In the NSTEMI group, the best cut-off point for MHR value determined as 2.33 with $56 \%$ sensitivity and $76 \%$ selectivity. TG/HDL-C ratio was found to be numerically higher in the disease group. However, it was not statistically significant $(p=0.386)$. The best cut-off point for the TG/HDL-C ratio determined as 3.33 , with $52 \%$ sensitivity and $54 \%$ selectivity. In the sub-group analyses performed with regards to coronary anatomy, a statistically significant relation was found between MHR and Circumflex (Cx) artery in predicting the lesion responsible for ischemia $(p=0.009)$. Conclusion In our study, MHR (OR=1.725, $p=0.030)$ was found to be statistically significant in NSTEMI patients, and although TG/HDL-C $(O R=1.526$, $\mathrm{p}=0.386$ ) ratio was numerically higher than the control group; it was not statistically significant in predicting severe coronary artery disease.
\end{abstract}

Keywords: Non-ST segment elevation acute coronary syndrome; Triglyceride/HDL cholesterol ratio; Coronary Artery Disease

\section{INTRODUCTION}

Acute myocardial infarct $(\mathrm{MI})$ is one of the most common causes of death in the world. $\mathrm{Ml}$ is a disease with high mortality and morbidity risk, and it is becoming more and more common. In recent years, many inflammation predictors discovered in patient groups with $\mathrm{Ml}$ diagnosis and studies are ongoing for new ones. Atherosclerosis is the basis of $\mathrm{Ml}$ and myocardial infarct is the most common atherosclerosis-associated cardiac artery disease (Calmarza, P., 2018). Factors of atherosclerosis development include hypertension, smoking, diabetes mellitus, gender, dyslipidemia, and genetics (Nijres, 2019; Vlad, 2019).

Also, parameters like concentrations of plasma triglyceride (TG) and triglyceride-rich lipoproteins (Silbernagel, 2019), apolipoprotein B (apoB) (Ooi, 2018), C-reactive protein (CRP) (Dittrich, 2019; Aksu, 2019) defined as risk determinants for 
coronary artery disease. Studies revealed relations between TG, monocyte, and HDL-C (Ganjali, 2017).

In their study, Hua-Yin et al. showed that the TG/HDL-C ratio could use as a cardiovascular risk factor indicator in adults $(\mathrm{Li}, \mathrm{H}, 2015)$. Dogan et. Al. Showed that $\mathrm{Tg} / \mathrm{HDL}$ ratio might be an essential predictor for an acute coronary syndrome in the young adult population (Dogan, C., 2019). Our study aimed to investigate the association of Triglyceride/HDL-C (TG/HDL-C) ratio, which is an indirect indicator of LDL particle size and can be easily calculated through complete blood count, with coronary artery disease in NSTEMI patients.

\section{MATERIALS AND METHODS Patient Characteristics}

This research is a retrospective study and consists of a total of 100 individuals who were older than 18 and younger than 80 years of age. Fifty of the patients diagnosed with NSTEMI and coronary angiography performed. The other half of the study population consists 50 individuals who had admitted to our cardiology outpatient clinic and had no acute coronary syndrome. Detailed anamnesis taken from both groups. In the angiography, the presence of $50 \%$ or more stenosis in at least one coronary considered as positive. Patients with heart attack history, who were diagnosed with moderate to severe valvular heart disease in echocardiography, patients with ventricle systolic dysfunction (ejection fraction $<0.40$ ), renal dysfunction (serum creatinine level $>1.5 \mathrm{mg} / \mathrm{dL}$ ), liver failure (liver function test results that are more than double the upper limits). Cancer patients, patients, acute or chronic infectious diseases, and patients using steroids excluded from the study. Hypertension (HT) defined as antihypertensive medication use or systolic blood pressure $>140 \mathrm{mmHg}$ and diastolic blood pressure $>90 \mathrm{mmHg}$. Diabetes mellitus (DM) defined as fasting blood glucose $\geq 126 \mathrm{mg} / \mathrm{dL}$ or use of anti-diabetic medication. Smoking was defined as regular tobacco use.

Ethics committee approval was not received as the study design was retrospective and written informed consent obtained from all patients before the procedure.

\section{Laboratory Measurements}

After an overnight fasting, the blood samples were taken from antecubital vein. Total and differential Monocyte counts measured by an automated hematology analyzer (Abbott Cell-Dyn 3700; Abbott Laboratory, Abbott Park, Illinois, USA).Absolute cell counts were used in the analyses. Total and high-density lipoprotein cholesterol (HDL), triglycerides, and fasting plasma glucose levels were measured using the Abbott Architect $\mathrm{C} 16000$ auto-analyzer (Architect $\mathrm{C} 16000$ autoanalyzer; Abbott Laboratory, Abbott Park, Illinois, USA).Plasma low-density lipoprotein cholesterol (LDL) concentrations were calculated using the Friedewald equation.MHR computed as monocyte count divided by HDL-C count.TG/HDL-C ratio computed as TG count divided by HDL-C count.

\section{Coronary Angiography}

Coronary angiography (CAG) performed by a femoral approach using the standard Judkins technique. Left and right coronary angiographies performed at various projections. The coronary arteries classified as normal based on a visual assessment in the absence of any luminal irregularities. According to findings on 
CAG, patients with a reduction of the diameter of the coronary artery by $\geq 50 \%$ diagnosed as having coronary heart disease (CHD).

\section{Statistical Analysis}

Statistical analysis was performed with SPSS 21.0 (IBM 1989, 2012) package program. The normality of distribution of the continuous variables was evaluated using the Kolmogorov-Smirnov test. Continuous data are expressed as mean \pm standard deviation and compared with Student's t-test. Correlation analyses were performed using Pearson's test. Receiver-operating characteristic (ROC) analysis was performed for the TG/HDL-C ratio to determine optimal cut-off values and to obtain the sensitivity and specificity of critical coronary artery stenosis. A p-value < 0.05 was considered statistically significant.

\section{RESULTS AND DISCUSSION}

Our study included a total of 100 patient (43 female and 57 male). Ages of the patients varied between 19 and 79 (57.6 \pm 13.2). NSTEMI groups are older than the control group presented in Table 1. Basic clinical and demographic characteristics and laboratory results of both groups shown in Table 1. There were no differences in the two groups for DM, HT, total cholesterol, triglyceride, creatinine, HDL-C, and LDL-C. The monocyte count was $0.68 \pm 0.03 \times 103 / \mu$ in the NSTEMI group. MHR was higher in the NSTEMI group $(p<0.05)$ (Table 2).

Monocyte count and TG/HDL-C ratio were higher in the NSTEMI group, but these variables did not reach statistical significance $(p=0,101 / p=0,386)$ (Table 2$)$. Based on regression analysis, the area under the curve is not predictive for the detection of lesions according to the ROC curve analysis used for determining severe coronary artery narrowness with TG/HDL-C. The area under the curve was $0.509(95 \% \mathrm{Cl}=0.395-0.623 ; \mathrm{p}=0.874)$ (Figure 1$)$. In sub-group analyses, MHR levels were found to be significantly higher in NSTEMI patients when the responsible lesion was the $\mathrm{Cx}$ artery ( $\mathrm{p}=0.009)$ (Figure 2$)$.

Table 1. Baseline clinical, demographic features and laboratory findings of the two groups

\begin{tabular}{llll}
\hline & \multicolumn{1}{c}{$\begin{array}{c}\text { NSTEMI group } \\
(\mathbf{n = 5 0 )}\end{array}$} & $\begin{array}{c}\text { Control group } \\
(\mathbf{n}=\mathbf{5 0})\end{array}$ & $\mathbf{p}$ \\
\hline Age, years (mean \pm SD) & $61.4 \pm 1.3$ & $53.8 \pm 2.1$ & 0,048 \\
Gender (female, male) & 13,37 & 30,20 & 0,001 \\
Tobacco use, (n) & 41 & 19 & 0,001 \\
Hypertension, (n) & 16 & 15 & 0,500 \\
Diabetes mellitus, (n) & 14 & 10 & 0,241 \\
Creatinine, mg/dL & $0.94 \pm 0.02$ & $0.78 \pm 0.01$ & 0,200 \\
Total plasma cholesterol, mg/dL & $204.76 \pm 10.63$ & $184.44 \pm 5.65$ & 0,260 \\
LDL-C, mg/dL & $138.57 \pm 6.39$ & $125.22 \pm 5.12$ & 0,307 \\
HDL-C, mg/dL & $43.20 \pm 8.77$ & $46.50 \pm 1.65$ & 0,174 \\
Triglycerides, mg/dL & $178.77 \pm 25.13$ & $170.84 \pm$ & 0,669 \\
\hline
\end{tabular}

NSTEMI: Non ST segment elevation myocardial infarction, HDL-C: High-density lipoprotein cholesterol, LDL-C: Low-density lipoprotein 
Table 2. Haematological and biochemical measurements of the two groups

\begin{tabular}{llll}
\hline & \multicolumn{1}{c}{$\begin{array}{c}\text { NSTEMI group } \\
(\mathbf{n}=5 \mathbf{0})\end{array}$} & $\begin{array}{c}\text { Control group } \\
(\mathbf{n}=\mathbf{5 0})\end{array}$ & \multicolumn{1}{c}{$\mathbf{p}$} \\
\hline Monocyte count $\left(10^{3} / \mathrm{mm}^{3}\right)$ & $0.68 \pm 0.03$ & $0.60 \pm 0.02$ & 0,101 \\
MHR & $172.25 \pm 84.17$ & $140.50 \pm 60.50$ & 0,030 \\
TG/HDL-C ratio & $5.40 \pm 1.53$ & $4.02 \pm 0.37$ & 0,386 \\
\hline
\end{tabular}

MHR: Monocyte/HDL-C ratio, NSTEMI: Non ST segment elevation myocardial infarction, TG/HDL-C ratio: Triglycerides/ HDL-C ratio

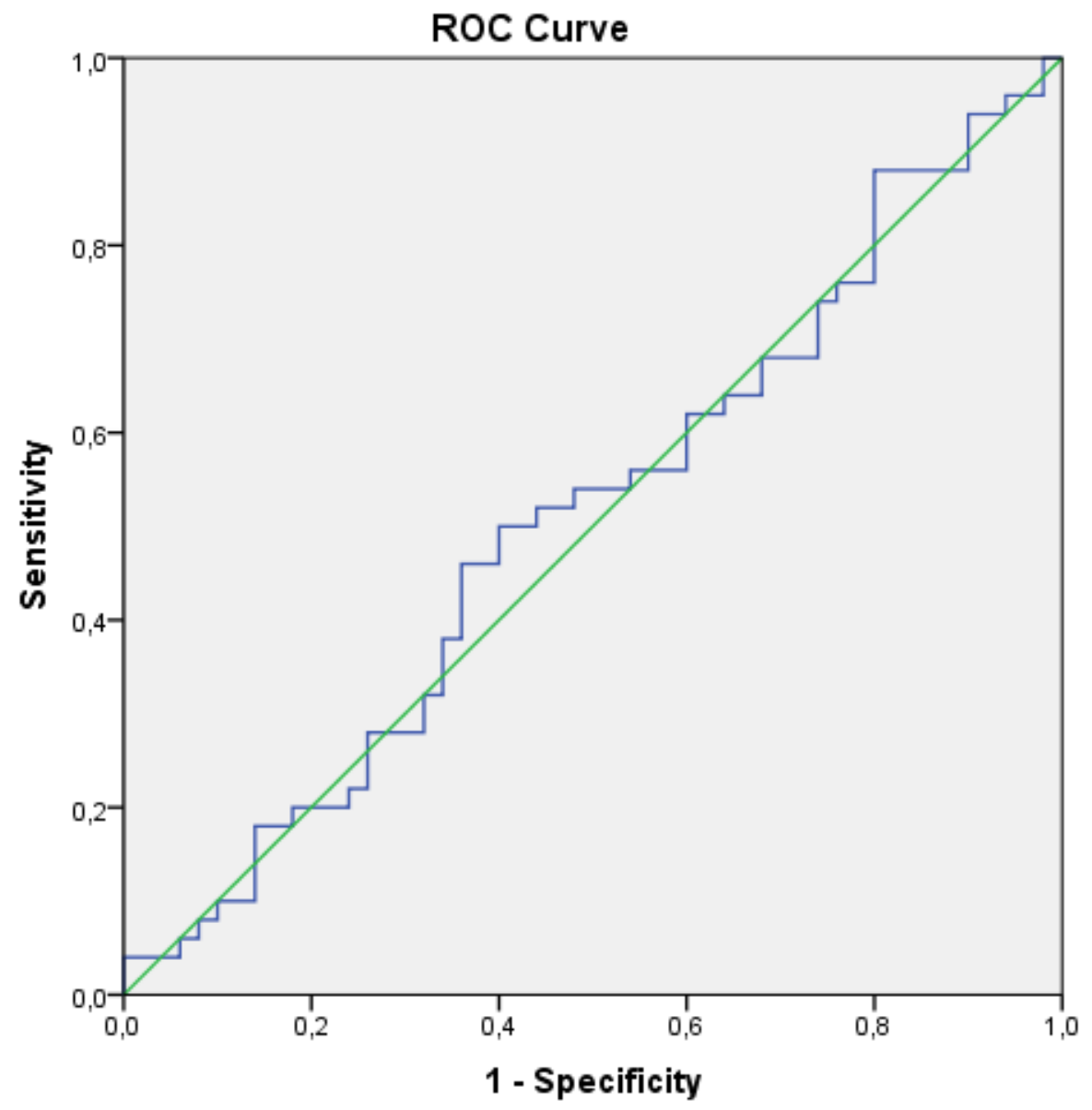

Figure 1: ROC analysis for determining the optimum cut-off TG/HDL-C ratio value for predicting critical coronary artery disease 




Figure 2: Relationship between the responsible coronary arteries lesion and TG/HDK-C ratio

In addition to conventional risk factors of atherosclerosis (smoking, hypertension, diabetes mellitus), there are studies with combined forms of lipoproteins such as TG/HDL-C, LDL-C/TG, LDL-C/HDL-C, TG/HDL-C, MHR (Zhao, X., 2018; Nakazato, J., 2019).

Although there is no consensus on the usability of the TG/HDL-C ratio, combined studies revealed that lipid indicators are more important for the determination of cardiovascular risk. Recent studies showed that small dense LDL and low HDL particles play a role as risk factors in atherosclerosis (Natarajan, P., 2019).

TG/HDL-C ratio, which has an active role in atherogenesis, is known to predict cardiovascular events in both healthy individuals and coronary artery patients (Yang, M., 2018; Owens, D., 2018).

In our study, MHR was statistically significantly higher in NSTEMI patient in comparison to the control group, and the best cut-off point for the TG/HDL-C ratio had $52 \%$ sensitivity and $54 \%$ selectivity.

Although the TG/HDL-C ratio was numerically higher than the control group, it was not statistically significant. The lack of knowledge of cut-off values for the TG/HDL-C ratio in our local population may be a factor. 
As the TG/HDL-C ratio can be crucial in atherosclerosis, it was numerically higher than the control group. However, it was not statistically significant. An impressive result in sub-group analyses showed a significant correlation with MHR when Cx artery was determined as the responsible coronary artery lesion in NSTEMI patients, although, there was no statistically significant correlation with TG/HDL-C ratio. Study Limitation is Few numbers of patients, single-center and retrospective study design are the limitations of our study.

\section{CONCLUSION}

In conclusion, as TG/HDL-C provides a simple, non-invasive, and fast result, large scale studies are required to standardize cut-off values to use in our society.

\section{REFERENCES}

Aksu, U., Gulcu, O., Aksakal, E., Kalkan, K., Öztürk, M., Korkmaz, A. F., . . . Demirelli, S. (2019). The association between CRP / Albumin ratio and in-stent restenosis development in patients with ST-segment elevation myocardial infarction. Journal of Clinical Laboratory Analysis, 33(4).

Calmarza, P., Lapresta, C., Martínez, M., Lahoz, R., \& Povar, J. (2018). Utility of myeloperoxidase in the differential diagnosis of acute coronary syndrome. Archivos De Cardiología De México, 88(5), 391-396.

Dittrich, J., Beutner, F., Teren, A., Thiery, J., Burkhardt, R., Scholz, M., \& Ceglarek, U. (2019). Plasma levels of apolipoproteins C-III, A-IV, and E are independently associated with stable atherosclerotic cardiovascular disease. Atherosclerosis, 281, 17-24.

Dogan, C., Bayram, Z., Karagoz, A., Bakal, R. B., Erdogan, E., Yilmaz, F., . . . Ozdemir, N. (2019). Is elevated triglyceride high density lipoprotein cholesterol ratio a risk factor that causes acute coronary syndrome to appear earlier? Bratislava Medical Journal, 119(12), 770-775.

Ganjali, S., Blesso, C. N., Banach, M., Pirro, M., Majeed, M., \& Sahebkar, A. (2017). Effects of curcumin on HDL functionality. Pharmacological Research, 119, 208218.

Li, H., Chen, B., Yang, Y., Ma, X., Liu, F., Fu, Z., . . Ma, Y. (2015). GW26-e2161 Optimal Cutoff of the Triglyceride to High-Density-Lipoprotein Cholesterol Ratio to Detect Cardiovascular Risk Factors Among Han Adults in Xinjiang. Journal of the American College of Cardiology, 66(16).

Nakazato, J., Hoshide, S., Wake, M., Miura, Y., Kuro-O, M., \& Kario, K. (2019). Association of calciprotein particles measured by a new method with coronary artery plaque in patients with coronary artery disease: A cross-sectional study. Journal of Cardiology.

Natarajan, P., Collier, T. S., Jin, Z., Lyass, A., Li, Y., Ibrahim, N. E., . . Januzzi, J. L. (2019). Association of an HDL Apolipoproteomic Score With Coronary Atherosclerosis and Cardiovascular Death. Journal of the American College of Cardiology, 73(17), 2135-2145.

Nijres, B. M., Samuel, B. P., \& Vettukattil, J. J. (2019). Subclinical atherosclerosis in patients with cyanotic congenital heart disease. International Journal of Cardiology, 282, 44.

Ooi, E. M., Ellis, K. L., Barrett, P. H., Watts, G. F., Hung, J., Beilby, J. P., . . . Mcquillan, B. M. (2018). Lipoprotein(a) and apolipoprotein(a) isoform size: Associations with angiographic extent and severity of coronary artery disease, and carotid artery plaque. Atherosclerosis, 275, 232-238. 
Owens, D., \& Tomkin, G. (2018). Commentary on High-density Lipoprotein Versus Low-density Lipoprotein Therapy and Cardiovascular Outcomes in Patients with Acute Coronary Syndromes by Nikolaos Papageorgiou et al. Current Cardiology Reviews, 14(4), 301-302.

Silbernagel, G., Scharnagl, H., Kleber, M. E., Delgado, G., Stojakovic, T., Laaksonen, R., . . . Grammer, T. B. (2019). LDL triglycerides, hepatic lipase activity, and coronary artery disease: An epidemiologic and Mendelian randomization study. Atherosclerosis, 282, 37-44.

Vlad, C., Burlacu, A., Florea, L., Artene, B., Badarau, S., Covic, A., . . Covic, A. (2019). A comprehensive review on apolipoproteins as nontraditional cardiovascular risk factors in end-stage renal disease: Current evidence and perspectives. International Urology and Nephrology.

Yang, M., Rigdon, J., \& Tsai, S. A. (2018). Association of triglyceride to HDL cholesterol ratio with cardiometabolic outcomes. Journal of Investigative Medicine, 67(3), 663-668.

Zhao, X., Sun, D., Xu, R., Guo, Y., Zhu, C., Wu, N., . . Li, J. (2018). Low-density lipoprotein-associated variables and the severity of coronary artery disease: An untreated Chinese cohort study. Biomarkers, 23(7), 647-653. 